\title{
Conexión entre centros de producción de ciencia y centros de producción de servicios tecnológicos: Visión desde Cuba y Perú
}

\author{
Connection between science production centers and technological \\ services production centers: Viev from Cuba and Peru
}

Recibido: marzo 08 de 2021 | Revisado: abril 02 de 2021 | Aceptado: abril 26 de 2021

\author{
Yadira Argota PÉRez \\ George Argota Pérez ${ }^{2}$
}

1 Empresa de Servicios Profesionales y Tecnológicos (ESAC). La Habana, Cuba. cm8lmp64064gmail.com

2 Centro de Investigaciones y Formación Superior en Educación, Salud y Medio Ambiente "AMTAWI". Perú. george.argota@gmail.com

\begin{abstract}
Resumen
El objetivo del estudio fue describir la conexión entre centros de producción de ciencia y centros de producción de servicios tecnológicos: una visión desde Cuba y Perú. Se indica una conexión social mediante preguntas de tipo gnoseológica, epistemológica, ontológica, ética y axiológica. Se establecen cinco indicadores de pensamiento estructural: 1 ro) estrategia, 2do) reconstrucción del conocimiento científico, 3ro), aplicación del conocimiento científico, 4to), función principal y 5to) desafíos. Se propone una pirámide de desempeño que muestra la visión, estrategia y voluntad de decisión y que vincula a centros de producción de ciencia y centros de producción de servicios tecnológicos para el desarrollo social. El planteamiento al tipo de pregunta permite el reconocimiento desde el origen y alcance de la conexión que se establece, su generación y validación como proceso vinculante, la práctica a desarrollar hasta el nivel de compromiso y responsabilidad que se asume para que el éxito como variable de satisfacción. Los indicadores de pensamiento estructural distinguen el resultado científico y las escalas de incrementos industriales lo que marcaron la correspondencia entre la trayectoria científica y especialización de la investigación con relación a la competencia industrial. Se concluye que, la descripción de la conexión entre los centros de producción de ciencia y los centros de producción de servicios tecnológicos desde una visión en Cuba y Perú permite generar innovaciones para la sostenibilidad local (comunidad) y su visibilidad como naciones en vía de desarrollo.
\end{abstract}

Palabras clave: conexión, impacto científico, productividad empresaria

\footnotetext{
Abstract

The aim of the study was to describe the connection between science production centers and technological
} contactara: revistacampus@usmp.pe. 
service production centers: a view from Cuba and Peru. A social connection is indicated through gnoseological, epistemological, ontological, ethical and axiological questions. Five indicators of structural thinking are established: 1st) strategy, 2nd) reconstruction of scientific knowledge, 3rd), application of scientific knowledge, 4th), main function and 5th) challenges. A performance pyramid is proposed that shows the vision, strategy and decision-making will and that links science production centers and technological services production centers for social development. The approach to the type of question allows the recognition from the origin and scope of the connection that is established, its generation and validation as a binding process, the practice to be developed up to the level of commitment and responsibility that is assumed so that success as a variable of satisfaction. The structural thinking indicators distinguish the scientific result and the scales of industrial increases, which marked the correspondence between the scientific trajectory and specialization of the research in relation to the industrial competence. It is concluded that the description of the connection between science production centers and technological services production centers from a perspective in Cuba and Peru allow the generation of innovations for local sustainability (community) and their visibility as nations on the way developing.

Key words: business productivity, connection, scientific impact

\section{Introducción}

En la actualidad, la comprensión de conexiones entre las organizaciones está determinado por el interés progresivo del modo de conocimiento en la ciencia, la tecnología e innovación (Borgatti \& Halgin, 2011; Parrilli \& Heras, 2016; Soderholm et al., 2019). No cabe duda, que la combinación del trabajo colaborativo aumenta las probabilidades hacia el éxito (Aalbers et al., 2016; Oh et al., 2016), por cuanto, todo resultado de conexiones entre las partes externas proporciona mayor acceso a recursos tangibles e intangibles como es, el conocimiento (Müller et al., 2018; Mun et al., 2019; Pancholi et al., 2019).

Ante la información que se presenta puede indicarse que el pensamiento de conexión ofrece múltiples y necesarios resultados, pero a criterios de los autores, algunos requerimientos deben prevalecer para dichas conexiones como los siguientes:

1. Mejoramiento reconocible de la imagen entre centros de producción (ej.: de ciencia y de servicios tecnológicos) para el desarrollo del talento humano 
2. Adecuación inmediata a los permanentes cambios y exigencias del entorno social

3. Capacidad diferenciada empresarial, a través de pensamientos innovativos

4. Marcaje de registros tecnológicos

5. Probabilidad elevada de mecanismos financieros y flujos económicos óptimos

6. Facilitación de cuotas de pagos por clientes de mercado

7. Viabilidad productiva y costo asequible

8. Comunicación direccionada, transparente y concisa

$\mathrm{Si}$ los criterios mencionados se tienen en cuenta, dejaría de entenderse la productividad exclusiva tradicional y entonces, se incorporarían valores agregados contemporáneos donde cualquier dispositivo de competencia comercial sería más viable (Dan, 2014; Javaid, 2014). Sin embargo, existe una función transcendental que debe planificarse y crearse desde la ciencia para crear conexiones no solo sociales, también lo es hacia el entorno y que signifique más relevancia (Toomey et al., 2020), aunque parece ser limitante para los países en vía de desarrollo.

En el caso de Cuba y Perú, la posibilidad de hacer ciencia como garantía y resguardo hacia los beneficios patrimoniales podría entenderse sobre la manera exponencial de innovar, aumentar las exportaciones, conectar las investigaciones a los factores económicos de la sociedad, generar resultados a corto plazo con bajo nivel de riesgo, vincularse hacia dispositivos de liderazgo, tener un pensamiento de ciclo cerrado de productos donde la conexión de la propia ciencia con los motores de desarrollo productivos locales debe permitir posicionar ambas naciones al reconocimiento internacional.

Entre diversos puntos de vista, el pensamiento estratégico como elemento propulsor verdadero para el logro de capacidades innovadoras convergentes y divergentes, indiscutiblemente estaría entre la relación indisoluble de los experimentos que se realizan y controlan por los centros de producción de ciencia y la disponibilidad tecnológica empresarial que existe en los centros de producción de servicios tecnológicos. Greig et al., (2019); Ma et al., (2020), mencionan que favorecer el desempeño en la viabilidad económica de las predicciones para aumentar los criterios de productividad, es una de las claves al desarrollo y para ello, debe considerarse pensar en sistemas de conexiones.

El objetivo del estudio fue describir la conexión entre centros de producción de ciencia y centros de producción de servicios tecnológicos: una visión desde Cuba y Perú.

\section{Método}

Se indicó una conexión social de pensamiento entre centros de producción de ciencia (CPC) y centros de producción de servicios tecnológicos (CPST) mediante preguntas (gnoseológica, epistemológica, ontológica, ética y axiológica). Asimismo, se establecieron cinco indicadores de pensamiento estructural: 1ro) estrategia, 2do) reconstrucción del conocimiento científico, 3ro) aplicación del conocimiento científico, 4to) función principal y 5to) desafíos.

Se consideró una pirámide de desempeño (visión, estrategia y voluntad 
de decisión) que vincula a centros de producción de ciencia y centros de producción de servicios tecnológicos para el desarrollo social.

De manera ética, se cumple con el deber de no conjeturar la realidad y el derecho de contribuir mediante el estado de opinión para el escrutinio científico.

\section{Resultados y Discusión}

La intención para el desarrollo de las localidades no es un fenómeno nuevo y, por el contrario, se persigue la búsqueda de mecanismos prospectivos para que los hallazgos en dos actores como son los centros de producción de ciencia y los centros de producción de servicios tecnológicos cumplan su responsabilidad y ganen protagonismo según las exigencias sociales.

La Tabla 1 muestra algunas preguntas de conexión entre un centro de producción de ciencia y un centro de producción de servicios tecnológicos.

Tabla 1

Conexión social de pensamiento / centro de producción de ciencia / centro de producción de servicios tecnológicos.

\begin{tabular}{ll}
\hline \multicolumn{1}{c}{ Tipo de pregunta } & \multicolumn{1}{c}{ Planteamiento } \\
\hline Gnoseológica & $\begin{array}{l}\text { ¿Cuántas unidades científico-técnicas de } \\
\text { centros de producción de servicios tecnoló- } \\
\text { gicos se asesoran por centros productores de } \\
\text { ciencia? } \\
\\
\text { ¿La validación de procesos en los centros } \\
\text { de producción de servicios tecnológicos se } \\
\text { establece desde el pensamiento científico de } \\
\text { los centros de producción de ciencia? } \\
\text { ¿Las comercializaciones de los centros de } \\
\text { producción de servicios tecnológicos se } \\
\text { sustentan en las prácticas experimentales de } \\
\text { los centros de producción de ciencia? } \\
\text { ¿Existe responsabilidad en los centros de } \\
\text { producción de ciencia y la economía de } \\
\text { competencia en los centros de producción de } \\
\text { servicios tecnológicos? } \\
\text { Ética } \\
\text { ¿Existe reconocimiento y compromiso en los } \\
\text { profesionales de los centros de producción } \\
\text { de ciencia con los orígenes y el desarrollo de } \\
\text { nuevos centros de producción de servicios } \\
\text { tecnológicos locales? }\end{array}$ \\
\hline Axiológica & \\
\hline &
\end{tabular}

La Tabla 2 muestra indicadores de pensamiento estructural de un centro de producción de la ciencia y un centro de producción de servicios tecnológicos. 
Tabla 2

Conexión social de pensamiento / centro de producción de ciencia / sector de servicios tecnológicos.

\begin{tabular}{|c|c|c|}
\hline $\begin{array}{l}\text { Indicadores de pensamiento } \\
\text { estructural }\end{array}$ & Centro producción de ciencia & $\begin{array}{l}\text { Centro de producción de servicios } \\
\text { tecnológicos }\end{array}$ \\
\hline Estrategia & Productividad puntual & Productividad sistemática \\
\hline $\begin{array}{l}\text { Reconstrucción del } \\
\text { conocimiento científico }\end{array}$ & Vanguardista & Tradicional - vanguardista \\
\hline $\begin{array}{l}\text { Aplicación del conocimiento } \\
\text { científico }\end{array}$ & Experimental & Industrial \\
\hline Función principal & $\begin{array}{l}\text { Social y de pensamiento cientí- } \\
\text { fico }\end{array}$ & Económico \\
\hline Desafíos & $\begin{array}{l}\text { Formación profesional, } \\
\text { Métodos de financiamiento, } \\
\text { Internacionalización global de la } \\
\text { ciencia }\end{array}$ & $\begin{array}{l}\text { Conexión con las universidades, } \\
\text { Centros de la ciencia, Instituciones } \\
\text { de ciencia, Internalización global de } \\
\text { la ciencia }\end{array}$ \\
\hline
\end{tabular}

La Figura 1 muestra la pirámide de desempeño que vincula el centro de producción de ciencia y el centro de producción de servicios tecnológicos para el desarrollo sostenible local (comunidad).

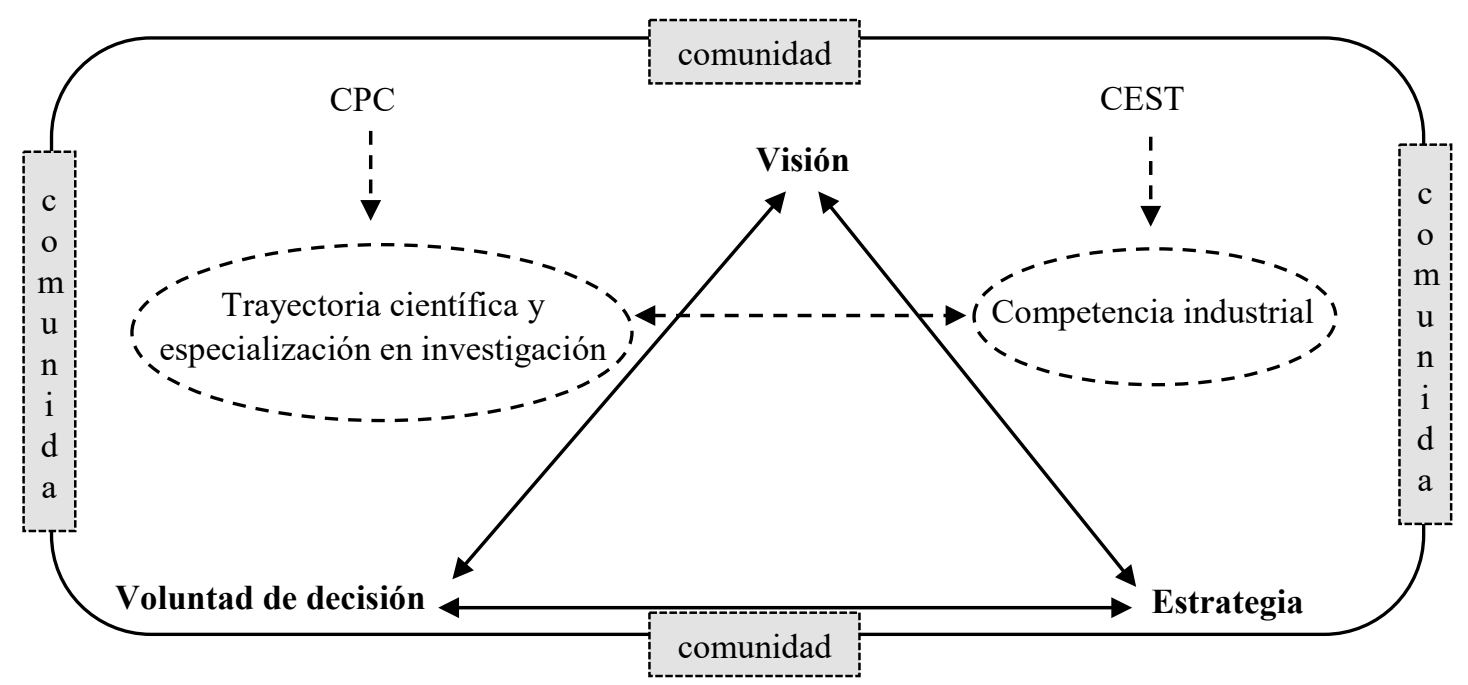

Figura 1. Pirámide de desempeño / centro de producción de ciencia / centro de producción de servicios tecnológicos / desarrollo sostenible local.

La incertidumbre humana probablemente, sea una condición para la búsqueda de alternativas de solución que estén orientadas a satisfacer las demandas sociales, pero jamás será suficiente con las pretensiones de los gobiernos en orientar políticas públicas que resulten distantes a las condiciones sociales. Son las propias condiciones sociales que determinan las políticas públicas a orientar por los gobiernos y, por tanto, debe fundamentarse toda estrategia de pensamiento para que exista conexión de requerimiento a favor de modificaciones progresivas bajo la dependencia de aquellos resultados que se logren.

Para indicar cualquier cambio, movimiento o transformación en el proceso de la cultura humana se necesita implementar métodos que no obedezcan a interpretaciones subjetivas $y$, por ende; 
al estancamiento. En este estudio, el planteamiento al tipo de pregunta permitió la caracterización entre la posible conexión social de los centros de producción de ciencia y los centros de producción de servicios tecnológicos donde se posibilita su reconocimiento desde el origen y alcance de la conexión que se establece, su generación y validación como proceso vinculante, la práctica a desarrollar hasta el nivel de compromiso y luego, la responsabilidad que se asume para que el éxito como variable de satisfacción.

Los indicadores de pensamiento estructural, si bien obedecen al tipo de centro de producción, pero lo distintivo estuvo en establecer un encadenamiento linealdel resultadocientíficocontodaescala de incrementos industriales a proponerse lo que marcaría la correspondencia entre la trayectoria científica y especialización de la investigación relacionada a la competencia industrial. Es decir, se propicia una relación directa de los centros de producción de ciencia y los centros de producción de servicios tecnológicos.
Cuba y Perú muestran diferencias considerables en todo orden de la sociedad. Sin embargo, el desarrollo para ambos países puede ser, desde la innegable relación que podría existir en el caso de Cuba y que denominan centros de estudios con los motores de desarrollo industrial y las universidades con los grupos de interés (ej.: sectores productivos locales) en el caso de Perú.

La conexión entre los CPC y CPST determina regularidades contributivas al pensamiento estratégico, mayores disponibilidades tecnológicas y métodos constructivos bidireccionales lo cual, estarían a favor de la innovación, su eficiencia y la visibilidad organizacional (Snyman \& Smallwood, 2017).

Se concluye que, la descripción de la conexión entre los centros de producción de ciencia y los centros de producción de servicios tecnológicos desde una visión en Cuba y Perú permite generar innovaciones para la sostenibilidad local (comunidad) y su visibilidad como naciones en vía de desarrollo.

\section{Referencias}

Aalbers, R., Dolfsma, W. \& Leenders, R. (2016). Vertical and horizontal cross-ties: benefits of crosshierarchy and cross-unit ties for innovative projects. J. Pro. Innov. Manag, 33, 141-153. https://doi. org/10.1111/jpim.12287

Borgatti, S.P. \& Halgin, D.S. (2011). On network theory. Org. Sci; 22(5), 1168-1181. https://doi. org/10.1287/orsc. 1100.0641

Greig, B., Nuthall, P. \& Old, K. (2019). Resilience and finances on Aotearoa
New Zealand farms: Evidence from a random survey on the sources and uses of debt. New Zealand Geographer; 75, 21-33. https://doi. org/10.1111/nzg. 12207

Ma, W., Bicknell, K. \& Renwick. A. (2020). Production intensification and animal health expenditure on dairy farms in New Zealand. J. Dairy Sci; 103, 1598-1607. https:// doi.org/10.3168/jds.2018-16039

Müller, J.M., Buliga, O. \& Voigt, K.I. (2018). Fortune favors the prepared: 
how SMEs approach business model innovations in Industry 4.0. Technol. Forcast. Soc. Change; 132, 2-17. https://doi.org/10.1016/j. techfore.2017.12.019

Mun, C., Kim, Y., Yoo, D., Yoon, S., Hyun, H., Raghavan, N. \& Park, H. (2019). Discovering business diversification opportunities using patent information and open innovation cases. Technol. Forcast. Soc. Change; 139, 144154. https://doi.org/10.1016/j. techfore.2018.11.006

Oh, D.S., Phillips, F., Park, S. \& Lee, E. (2016). Innovation ecosystems: a critical examination. Technovation; 54, 1-6. https://doi.org/10.1016/j. technovation.2016.02.004

Pancholi, S., Yigitcanlar, T. \& Guaralda, M. (2019). Place making for innovation and knowledgeintensive activities: the Australian experience. Technol. Forcast. Soc. Change; 146, 616-625. https://doi.org/10.1016/j. techfore.2017.09.014

Parrilli, M.D. \& Heras, H.A. (2016). STI and DUI innovation modes: scientific-technological and context-specific nuances. Res. Pol; 45(4), 747-756. https://doi. org/10.1016/j.respol.2016.01.001

Snyman, T. \& Smallwood, J. (2017). Improving productivity in the business of construction. Procedia Engineering; 182, 651657. https://doi.org/10.1016/j. proeng.2017.03.175

Soderholm, P., Hellsmark, H., Frishammar, J., Hansson, J., Mossberg, J. \& Sandstrom, A. (2019). Technological development for sustainability: the role of network management in the innovation policy mix. Technol. Forcast. Soc. Change; 138, 309323. https://doi.org/10.1016/j. techfore.2018.10.010

Toomey,A.H., Strehlau, H.L., Manzolillo, B. \& Thomas, C. (2020). The place-making potential of citizen science: Creating social-ecological connections in an urbanized world. Landscape and Urban Planning; 200, 1-8. https://doi.org/10.1016/j. landurbplan.2020.103824 
